\title{
AVALIAÇÃO DA RELAÇÃO ENTRE AS DIMENSÕES DA ÓRBITA E DO FORAME ÓPTICO COM AS DIMENSÕES DO NERVO ÓPTICO PELA TOMOGRAFIA COMPUTADORIZADA HELICOIDAL
}

\author{
Priscila Alves NASCIMENTO' \\ Samira HENRIQUE \\ Artur Hess NETO3 \\ Bruna Vargas OLIVIERI ${ }^{4}$ \\ Rodrigo de Andrade RUFINO ${ }^{5}$ \\ Maria José APS TUCUNDUVA 6
}

\section{RESUMO}

A órbita é uma escavação óssea no crânio, que tem função de apoio e proteção ao bulbo do olho, seus vasos e nervos. Ela é uma pirâmide óssea composta por 7 ossos que compõem quatro paredes: um teto, uma base, parede lateral e medial; são eles o osso esfenoidal, etmoide, maxila, palatino, placa orbital do osso frontal, zigomático e lacrimal. O nervo óptico é composto pelas células ganglionares da retina e comunica o globo ocular ao cérebro. Pode ser dividido em 4 partes que possuem comprimentos diferentes, num total de $50 \mathrm{~mm}$, divididos em partes intraocular, intraorbital, intracanalicular e intracraniano. Tanto a órbita quanto o nervo óptico devem estar dentro dos parâmetros anatômicos de normalidade e qualquer alteração nesse padrão é uma possível causa de perda ou diminuição da acuidade visual. Uma dessas possíveis causas da diminuição da acuidade poderia ser uma anormalidade anatômica dos ossos orbitais, causando uma diminuição ou distorção do espaço orbital. Outra hipótese pode ser uma desproporção entre as dimensões do nervo óptico e o forame óptico, causando uma compressão e consequente lesão nervosa, ou até mesmo a existência de uma desproporção entre a cavidade orbital e os diâmetros do nervo óptico. Para compreender a relação entre a perda da acuidade visual e as alterações anatômicas citadas é essencial estabelecer o padrão de normalidade da órbita e do nervo óptico e suas dimensões. Para o tipo de análise proposta, a tomografia computadorizada helicoidal pode fornecer esses padrões de normalidade. Com esse intuito, foram observados 50 exames em cortes axiais representando a cavidade orbital, o canal óptico e o nervo óptico que foram identificados e mensurados pelo programa OsiriX. O comprimento da cavidade da órbita apontou para o lado direito, média de 39,40 mm e, lado esquerdo, média de 38,40 mm. O comprimento da parte intraorbital do nervo óptico para o olho direito, média $23,90 \mathrm{~mm}$ e o olho esquerdo, média de $23,60 \mathrm{~mm}$. O diâmetro do canal óptico do lado direito alcançou um valor médio de 4,900mm e do lado esquerdo obteve uma média de $4,290 \mathrm{~mm}$.

1 Graduanda do curso de medicina na Universidade Cidade de São Paulo. Ingresso em 2010. Conclusão em 2015.

2 Graduanda do curso de medicina na Universidade Cidade de São Paulo. Ingresso em 2014. Conclusão em 2019.

3 Graduado em engenharia metalúrgica pelo Centro Universitário da FEI no período de 2002 a 2006. Graduando do curso de medicina na Universidade Cidade de São Paulo. Ingresso em 2010. Conclusão em 2015

4 Graduanda do curso de medicina na Universidade Cidade de São Paulo. Ingresso em 2014. Conclusão em 2019.

5 Graduada em odontologia pela Universidade Cidade de São Paulo. Doutorado em diagnóstico bucal pela faculdade de odontologia da USP. Professora dos cursos de medicina e biomedicina na Universidade Cidade de São Paulo.

6 Graduado em biomedicina pela Universidade Cidade de São Paulo. Mestre em ciências morfofuncionais pelo departamento de anatomia do instituto de ciências biomédicas da USP. Professor do curso de Biomedicina na Universidade Cidade de São Paulo. 
NASCIMENTO PA, HENRIQUE S, HESS NETOA, OLIVIERI BV, RUFINO RA, TUCUNDUVA MJAPS. Avaliação da relação entre as dimensões da órbita e do forame óptico com as dimensões do nervo óptico pela tomografia computadorizada helicoidal. Rev. Odontol. Univ. Cid. São Paulo 2018 jan-mar; 30(1):54-63

ISSN 1983-5183

Por meio da análise do presente estudo foi possível estabelecer valores numéricos para o padrão de normalidade, permitindo investigações causais posteriores.

PALAVRAS-CHAVE: ÓRBITA - NERVO ÓPTICO - TOMOGRAFIA COMPUTADORIZADA ESPIRAL

\title{
EVALUATION OF THE RELATION BETWEEN THE ORBIT AND THE OPTIC FORAMEN DIMENSIONS WITH THE OPTIC NERVE DIMENSIONS BY HELICAL COMPUTED TOMOGRAPHY
}

\begin{abstract}
The orbit is a bone digging in the skull, which has a supporting and protective function to the eye bulb, its vessels and nerves. It is a bone pyramid composed of 7 bones that consists of four walls: a ceiling, a base, lateral and medial wall. They are the sphenoid bone, ethmoid, maxilla, palatine, orbital plaque of the frontal bone, zygomatic and lacrimal. The optic nerve is composed of the ganglion cells of the retina, and they communicate the ocular globe to the cortex. It can be divided into 4 parts that present different lengths, being a total of $50 \mathrm{~mm}$, divided into intraocular, intraorbital, intracanalicular and intracranial parts. Both the orbit and the optic nerve must be within the anatomical parameters of normality, and any change in this pattern is a possible cause of loss or decrease of visual acuity. One of these possible causes of decreased acuity could be an anatomical abnormality of the orbital bones, causing a decrease or distortion of the orbital space. Another hypothesis may be a disproportion between the dimensions of the optic nerve and the optic foramen, causing a compression and consequent nerve damage, or even the existence of a disproportion between the orbital cavity and the diameters of the optic nerve. To understand the relationship between loss of visual acuity and the anatomical changes cited, it is essential to establish the normality of the orbit and optic nerve and its dimensions. For analysis proposed, helical computerized tomography can provide these normality patterns. For this purpose, 50 exams were performed on axial sections representing the orbital cavity, optic canal and optic nerve that were identified and measured by the OsiriX program. The cavity length of the orbit was to the right side, mean of $39.40 \mathrm{~mm}$ and left side: mean of $38.40 \mathrm{~mm}$. The length of the intraorbital portion of the optic nerve to the right eye, mean $23.90 \mathrm{~mm}$ and the left eye, mean of $23.60 \mathrm{~mm}$. The diameter of the optical channel on the right side reached an average value of $4.900 \mathrm{~mm}$ and the left side obtained an average of $4.290 \mathrm{~mm}$. Through the analysis of the present study it was possible to establish numerical values for the normality pattern allowing further causal investigations.
\end{abstract}

KEY WORDS: ORBIT - OPTIC NERVE - TOMOGRAPHY, SPIRAL COMPUTED

\section{INTRODUÇÃO}

A cavidade orbital é uma escavação óssea essencial para alojamento e proteção do globo ocular, do nervo óptico e de muitas estruturas que entram na cavidade a partir da fissura orbital superior, compreendendo em sua porção superior os nervos troclear, frontal e lacrimal e a veia oftálmica superior, e em sua porção inferior as divisões superior e inferior do nervo oculomotor, o abducente, o nasociliar e fibras simpáticas ${ }^{1,2,3}$. Devido à importância dessas estruturas para o funcionamento perfeito do globo ocular, a integridade dessa cavidade é essencial para o olho exercer sua capacidade visual máxima, permitindo assim o sentido da visão, essencial para a manutenção de uma vida saudável, tanto psicológica quanto fisiologicamente. 
NASCIMENTO PA, HENRIQUE S, HESS NETOA, OLIVIERI BV, RUFINO RA, TUCUNDUVA MJAPS. Avaliação da relação entre as dimensões da órbita e do forame óptico com as dimensões do nervo óptico pela tomografia computadorizada helicoidal. Rev. Odontol. Univ. Cid. São Paulo 2018 jan-mar; 30(1):54-63

ISSN 1983-5183

Muitas doenças podem acometer estruturas ósseas da cavidade orbital ou qualquer um de seus componentes internos ${ }^{4,5,6}$. Exemplos de doenças seriam o meningeoma, acometendo o nervo óptico ${ }^{7,8}, 0$ glioma ${ }^{9}$ a displasia fibrosa óssea que acomete a própria estrutura óssea ${ }^{10,11}$, metástases de câncer de mama ${ }^{5}$ ${ }^{6}$ oftalmopatia de Graves ${ }^{2}$, entre outras. A detecção precoce de doenças e seu manejo adequado podem amenizar os sintomas da perda visual causados por essas doenças ou até mesmo preveni-los.

Existem muitos métodos imaginológicos que avaliam as estruturas da cavidade orbital. O utilizado neste trabalho foi a tomografia computadorizada helicoidal, pois o uso dela permite uma avaliação nítida da estrutura óssea da órbita dessas estruturas moles ao redor, podendo identificar qualquer alteração que possa ser sugestiva de anormalidade ${ }^{12,13}$.

Em relação à avaliação das imagens na tomografia computadorizada helicoidal, existe uma escala onde -1000 é o tom mais escuro, o 0 é um médio e +1000 é um tom claro. Uma escala de cinzas foi criada especialmente para a tomografia computadorizada e sua unidade foi chamada de unidade Hounsfield (HU). Sendo zero unidades Housfield (0 HU) a água, o ar -1000 (HU), o osso 300 a 350 HU, a gordura de -120 a -80 HU e por fim o músculo de 50 a 55 HU12, 13, 14.

\section{Objetivo}

Estudar, por meio da tomografia computadorizada helicoidal, as dimensões da cavidade orbital, do canal óptico e do nervo óptico, visando estabelecer um padrão de normalidade individual e entre essas estruturas, que permita compreender a relação entre a perda da acuidade visual e as alterações anatômicas posteriormente.

\section{Material e Métodos}

Foram observados 50 exames de tomografias computadorizadas helicoidais, pertencentes a um banco de dados de pacientes com solicitação de exame da face, que foi disponibilizado para uso neste trabalho de Iniciação Científica, constituindo, assim, uma amostra de conveniência, mediante aprovação do CEP desta instituição, sob o número de aprovação 30903314.3.0000.0064.

Foram observados cortes axiais nos quais a cavidade orbital, o canal óptico e o nervo óptico foram identificados e mensuraram-se os comprimentos da cavidade orbital e do nervo óptico, o diâmetro do canal óptico e do nervo óptico. A mensuração foi realizada pelo programa OsiriX. Foi calculada a relação entre os comprimentos do nervo óptico e da cavidade orbital a partir das medidas obtidas separadamente.

Para a análise estatística dos dados, utilizamos a análise de variância (ANOVA) não paramétrica (U de Mann-Whitney). Todos os resultados foram expressos em desvio-padrão, onde foi considerado significante sempre que $p<0,05$. Para o respectivo tratamento estatístico, utilizamos o software computacional "GraphPadPrism" versão 5.03.

\section{Resultados}

O presente trabalho consistiu na análise de 50 tomografias computadorizadas helicoidais, de ambos os sexos, todas em corte axial. Os parâmetros estudados foram comprimento da cavidade orbital, comprimento do nervo óptico, relação entre os comprimentos do nervo óptico e da cavidade orbital, diâmetro do nervo óptico e diâmetro do canal óptico. 


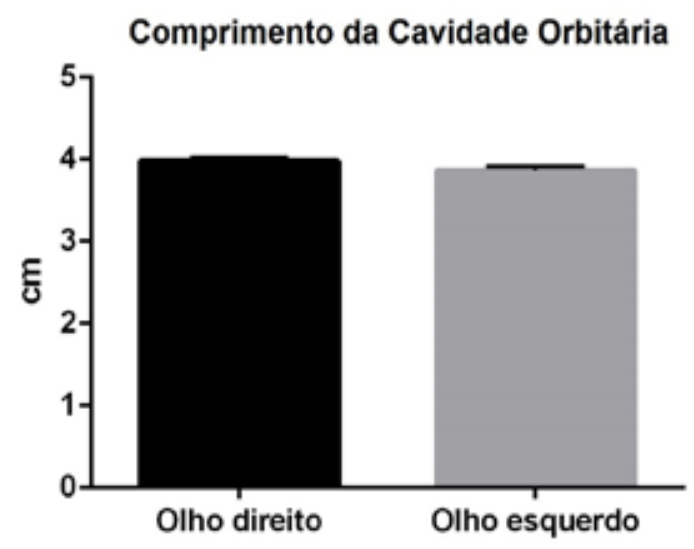

Figura 1: Comparação do comprimento da cavidade orbital do olho direito e esquerdo.

Para se obter o comprimento da cavidade orbital, a partir das bordas externas da mesma, foi traçada uma linha látero-medial, a partir da qual traçou-se uma reta perpendicular a esta, até atingir o canal óptico. O valor da perpendicular representa o comprimento da cavidade orbital (Figura 1). Foram encontrados os seguintes valores: olho direito, média de 39,40mm (desvio-padrão 0,3343), sendo o valor máximo encontrado de 48,90mm e mínimo de 33,50 mm; olho esquerdo: média de 38,40mm (desvio-padrão 0,3136), sendo o valor máximo 46,40mm e mínimo 33,20mm.

Os valores encontrados para o comprimento da parte intraorbital do nervo óptico (Figura 2) foram: para o olho direito, média de 23,90mm (desvio-padrão 0,2948), sendo o máximo atingido de 29,70mm e mínimo de 17,40mm; para o olho esquerdo, média de 23,60mm, máximo de 29,60mm e mínimo de 17,90mm (desvio-padrão 0,3029). Esses dados foram coletados a partir da subtração do comprimento da cavidade orbital de uma nova perpendicular traçada até a parte posterior do bulbo do olho.

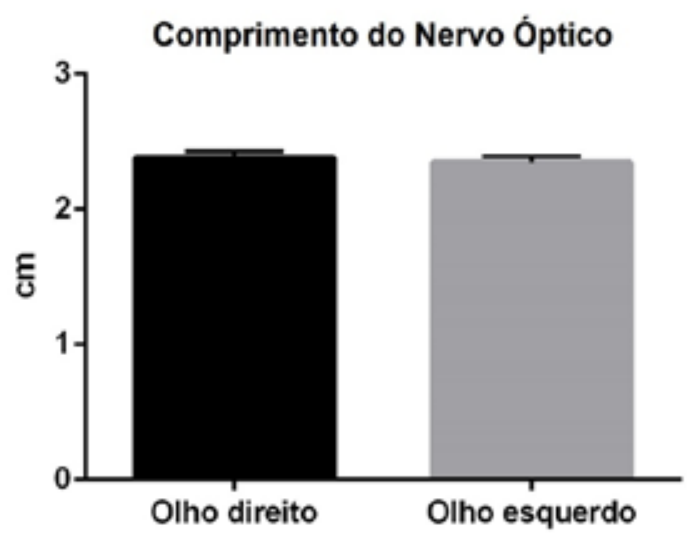

Figura 2: Comparação do comprimento do nervo óptico do olho direito e esquerdo.

Ao relacionar o comprimento do nervo óptico com o comprimento da cavidade orbital, obtivemos uma proporção média de 60,2\% no olho direito (desvio-padrão 5,585), sendo a maior proporção encontrada 
NASCIMENTO PA, HENRIQUE S, HESS NETOA, OLIVIERI BV, RUFINO RA, TUCUNDUVA MJAPS. Avaliação da relação entre as dimensões da órbita e do forame óptico com as dimensões do nervo óptico pela tomografia computadorizada helicoidal. Rev. Odontol. Univ. Cid. São Paulo 2018 jan-mar; 30(1):54-63

\section{ISSN 1983-5183}

de $70,70 \%$ e a menor de 48,40\%. Essa relação média no olho esquerdo foi de $61,45 \%$ (desvio-padrão 5,779), variando de $71,10 \%$ a $48 \%$.

No parâmetro diâmetro do nervo óptico, foi utilizado para sua medição o maior diâmetro do nervo óptico, entre toda sua extensão e entre todos os cortes de TC (Figura 3). No olho direito, obtivemos um valor médio de 5,500mm (desvio-padrão 1,134), com variação entre 7,610 mm e 2,900mm. Já no olho esquerdo o valor médio foi, coincidentemente, igual ao olho direito, 5,500 mm (desvio-padrão 1,016), variando entre $7,470 \mathrm{~mm}$ e $2,710 \mathrm{~mm}$.

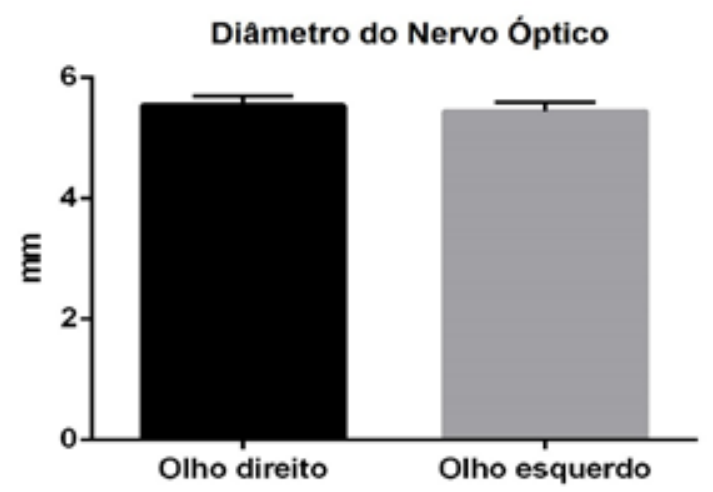

Figura 3: Comparação do diâmetro do nervo óptico do olho direito e esquerdo.

O último parâmetro analisado foi o diâmetro do canal óptico (Figura 4). Obteve-se a medida traçandose uma linha látero-medial nos dois pontos mais altos da TC, em que foi observada a maior largura. No olho direito alcançou um valor médio de 4,900 mm (desvio-padrão 1,385), com valor máximo de 6,840mm e mínimo de 1,710mm. O olho esquerdo obteve uma média de 4,290mm (desvio-padrão 1,585), sendo o máximo encontrado de 7,760mm e o mínimo de 1,550mm.

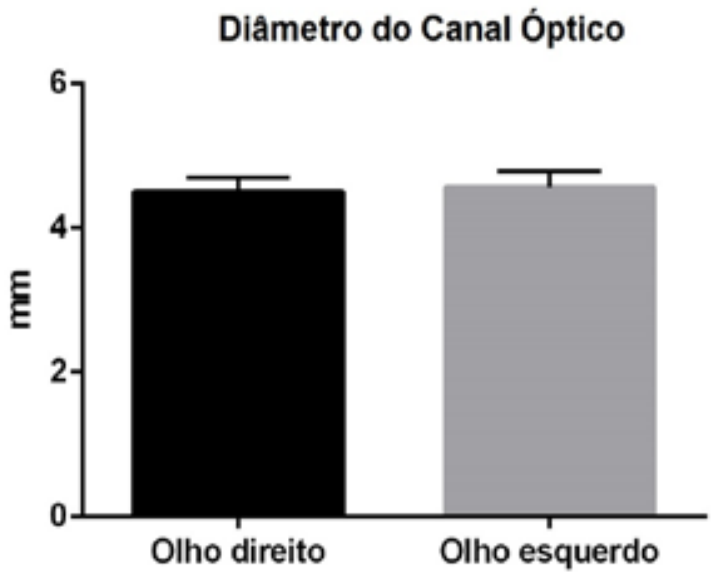

Figura 4: Comparação do diâmetro do canal óptico do olho direito e esquerdo. 


\section{DISCUSSÃO}

Podemos relacionar os valores encontrados neste estudo com alguns dados encontrados na literatura sobre essa região anatômica. De acordo com Kanski ${ }^{15}$ (2008) e também com estudo realizado por Guimarães $^{16}(2003)$, a parte intraorbital do nervo óptico varia de 25 a $30 \mathrm{~mm}$ de comprimento. No presente trabalho, os valores obtidos variaram de $17,40 \mathrm{~mm}$ e $29,70 \mathrm{~mm}$. Podemos observar que o valor máximo obtido corresponde ao limite superior encontrado na literatura, porém o limite inferior obtido tem uma variação de 7,6mm para menos. Algumas hipóteses podem ser aventadas para tal achado. Pode decorrer simplesmente de uma variação anatômica fisiológica, por terem os estudos sido realizados em populações e em localidades diferentes, ou também pode ser devido à variação de idade da população estudada nos diversos trabalhos. Porém, o valor médio encontrado neste trabalho foi de $23,90 \mathrm{~mm}$ no olho direito e de $23,60 \mathrm{~mm}$ no olho esquerdo; portanto, se considerarmos que foram só alguns indivíduos que desviaram o limite inferior para baixo do encontrado na literatura, cria-se a hipótese de que esses pacientes com nervo óptico mais curto possam ter alguma patologia que gere esse achado, enquanto os valores médios representam um padrão de normalidade.

Já o diâmetro do canal óptico, na literatura mede aproximadamente 5-6mm de acordo com Ferreira e Santos ${ }^{17}$ (2012). Neste estudo, encontramos valores entre 1,550mm e 7,760mm. No estudo realizado por Liu et al. ${ }^{18}$ (2000), em cortes axiais e coronais de tomografia computadorizada, encontrou-se para o diâmetro do canal óptico um valor médio de $3.57+/-0.61 \mathrm{~mm}$. O raciocínio desse achado, sendo tanto o limite inferior quanto o superior maiores do que os encontrados na literatura, pode ser o mesmo do que o do comprimento do nervo óptico e, nessa situação, que pode gerar assunto para outro estudo, há a possibilidade de esses indivíduos, que têm o diâmetro do nervo óptico menor, serem portadores de baixa acuidade visual.

A relação entre o comprimento do nervo óptico e o comprimento da cavidade orbital é uma medida da qual, até o momento, não encontramos dados comparativos na literatura. Portanto, esse achado, de um valor médio de $60,2 \%$ no olho direito e de $61,45 \%$ no esquerdo, pode representar um padrão de normalidade dessa relação. $O$ valor do comprimento da cavidade orbital encontrado pode também ser adotado como um valor normal para a população dessa região demográfica.

O valor médio do diâmetro do nervo óptico intraorbital encontrado neste estudo foi de 5,500 $\mathrm{mm}$ em ambos os olhos e, comparando-se com os dados encontrados na literatura, onde temos um valor médio de 3 a $4 \mathrm{~mm}$ de acordo com Kanski15 (2008), observamos que há pouca diferença entre os achados; levandose em consideração que, novamente, a população estudada pode ter diferença significativa de idade e de localização geográfica, os valores encontrados por nós neste trabalho podem ter significância como padrão populacional, tratando-se de um estudo da população brasileira. 
NASCIMENTO PA, HENRIQUE S, HESS NETOA, OLIVIERI BV, RUFINO RA, TUCUNDUVA MJAPS. Avaliação da relação entre as dimensões da órbita e do forame óptico com as dimensões do nervo óptico pela tomografia computadorizada helicoidal. Rev. Odontol. Univ. Cid. São Paulo 2018 jan-mar; 30(1):54-63

\section{ISSN 1983-5183}

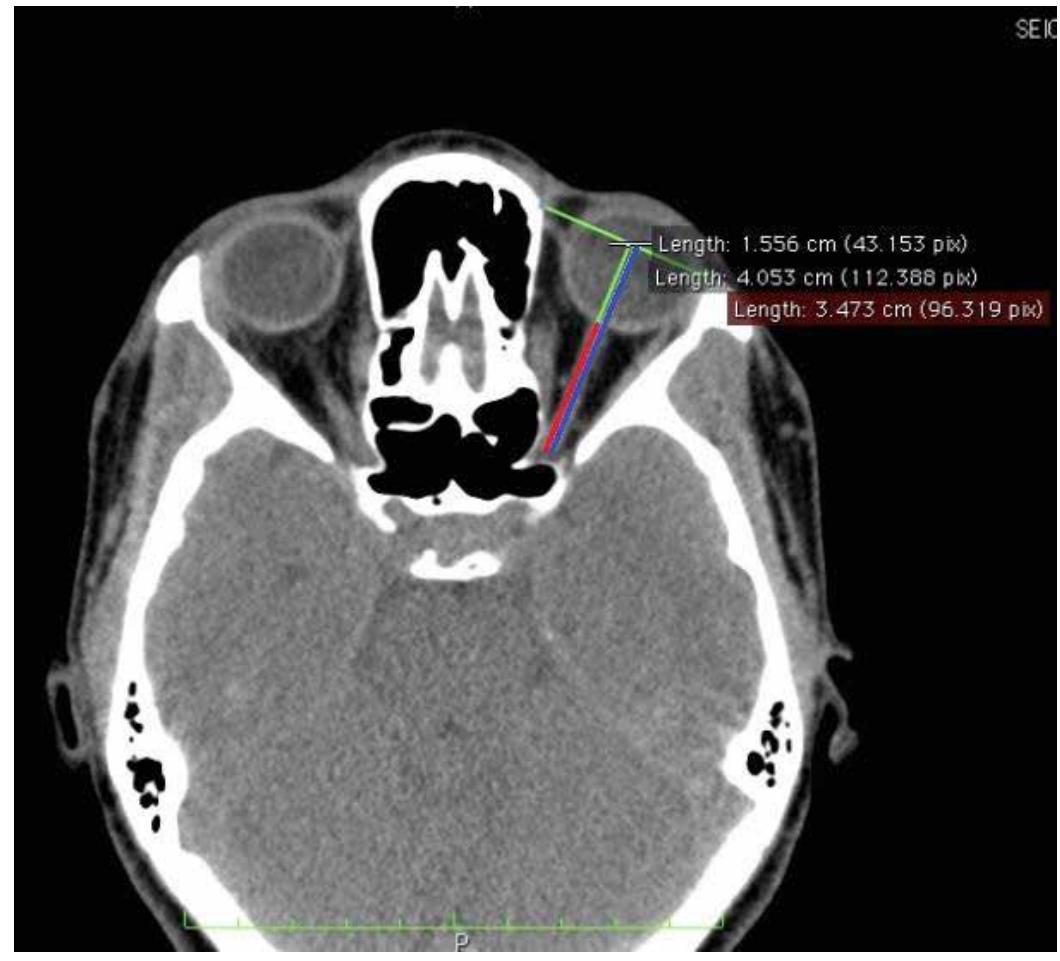

Figura 5. Corte axial de TC em que está presente o comprimento da cavidade orbital (linha azul) e o comprimento do nervo óptico (linha vermelha).

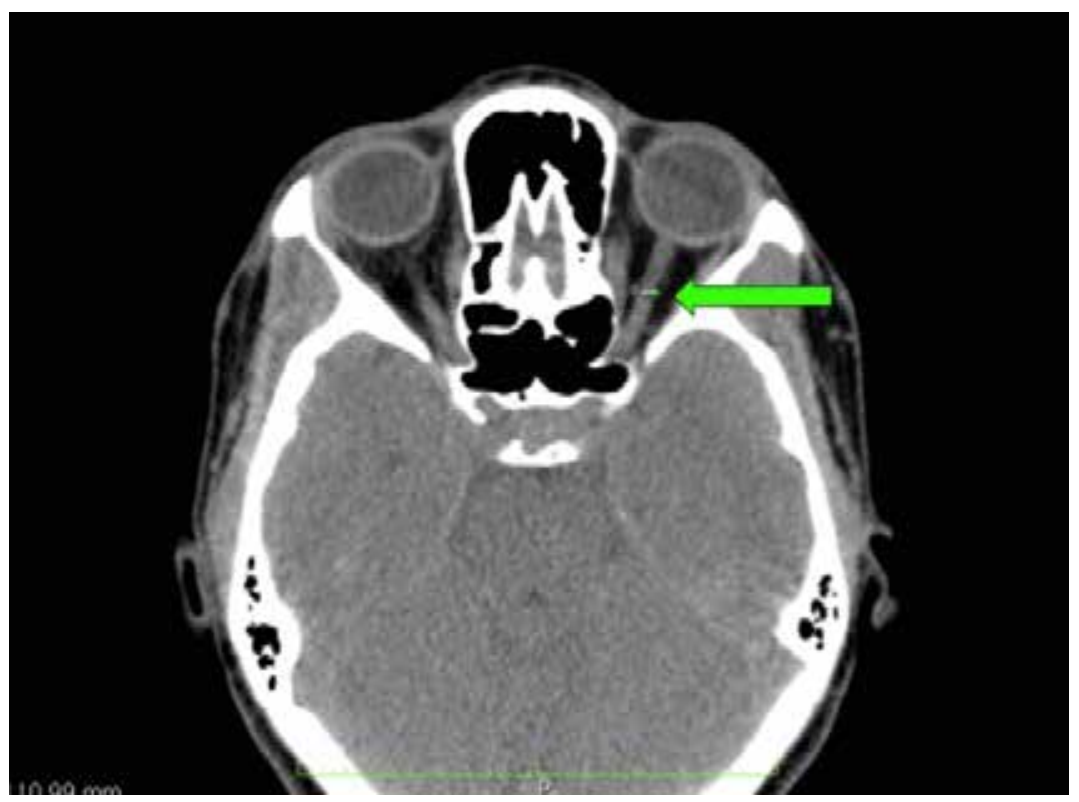

Figura 6. Corte axial de TC em que está presente o diâmetro da parte intraorbital do nervo óptico. 


\section{ISSN 1983-5183}

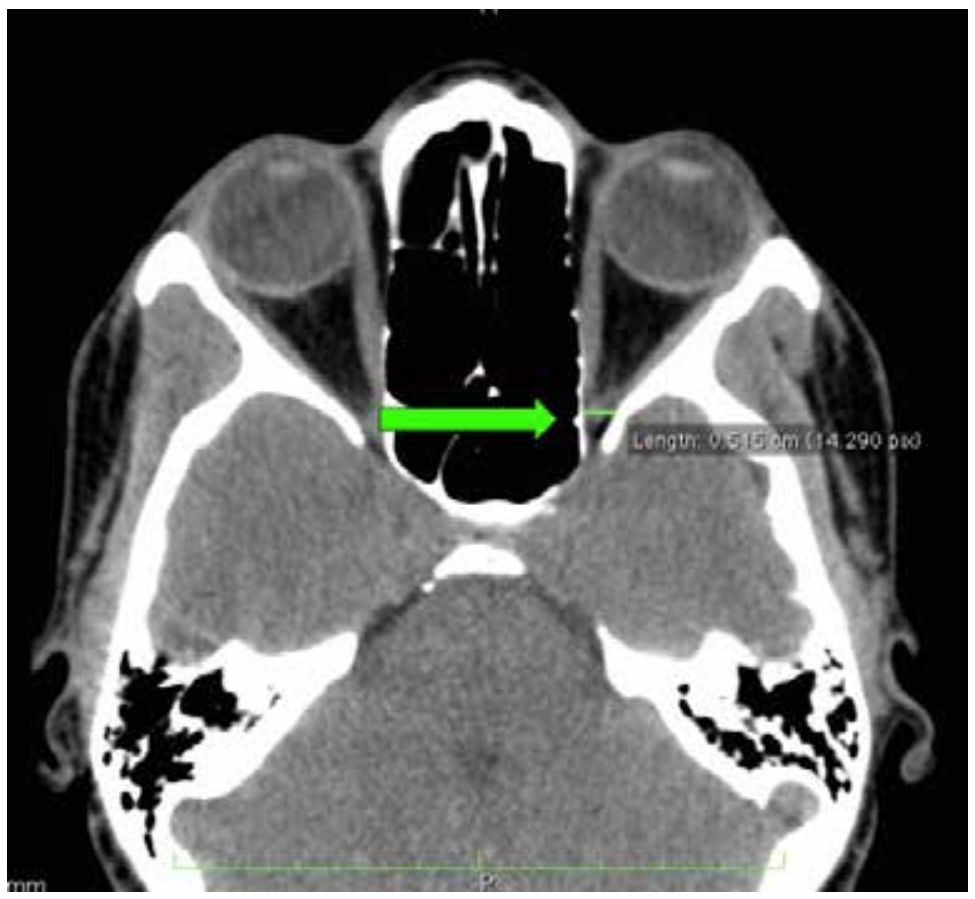

Figura 7. Corte axial de TC em que está presente o diâmetro do canal óptico.

\section{CONCLUSÃO}

Por meio deste estudo concluímos que o comprimento da cavidade da órbita apontou, para o lado direito, média de $39,40 \mathrm{~mm}$ e, lado esquerdo, média de $38,40 \mathrm{~mm}$. 0 comprimento da parte intraorbital do nervo óptico para o olho direito, média $23,90 \mathrm{~mm}$ e o olho esquerdo, média de $23,60 \mathrm{~mm}$. 0 diâmetro do canal óptico do lado direito alcançou um valor médio de $4,900 \mathrm{~mm}$ e do lado esquerdo obteve uma média de $4,290 \mathrm{~mm}$.

A partir dos estudos utilizados para realizar a revisão de literatura, pode-se observar que existem poucos dados em relação ao padrão de normalidade das estruturas estudadas no presente projeto. As poucas informações obtidas foram de autores e pesquisadores internacionais, o que pode variar muito se comparado aos valores obtidos aqui no Brasil. Portanto, com os números encontrados neste estudo, podemos traçar um padrão de normalidade dentro da população brasileira e abrir discussões e mais estudos para corroborar nossos achados. 


\section{REFERÊNCIAS}

1. TESTUT L, Latarjet A. Tratado de anatomia humana. Barcelona: Salvat; 1979.

2. MOORE KL. Anatomia orientada para a clínica. 4. ed. Rio de Janeiro: Elsevier; 2001.

3. RENE C. Update on orbital anatomy. Eye (London, England) 2006 Oct;20(10):1119-29.

4. DUMONT AS, Boulos PT, Jane JA, Jr., Ellegala DB, Newman SA, Jane JA, Sr. Cranioorbital fibrous dysplasia: with emphasis on visual impairment and current surgical management. Neurosurgical focus 2001 May 15;10(5):E6.

5. MILLER NR. Primary tumours of the optic nerve and its sheath. Eye (London, England) 2004 Nov;18(11):1026-37.

6. KHAN SN, Sepahdari AR. Orbital masses: CT and MRI of common vascular lesions, benign tumors, and malignancies. Saudi J Ophthalmol 2012 26(4):373-83.

7. DUTTON JJ. Optic nerve sheath meningiomas. Survey of ophthalmology 1992 Nov-Dec;37(3):167-83.

8. BROWER JV, Amdur RJ, Kirwan J, Mendenhall WM, Friedman W. Radiation therapy for optic nerve sheath meningioma. Practical radiation oncology 2013 Jul-Sep;3(3):223-8.

9. NIYAZI M, Karin I, Sohn M, Nachbichler SB, Lang P, Belka C, et al. Analysis of equivalent uniform dose (EUD) and conventional radiation treatment parameters after primary and re-irradiation of malignant glioma. Radiation oncology (London, England) 2013 Dec $8(1): 287$.

10. SANTOS JN, Vieira TSLS, Góis Filho DM, Vasconcelos SJA, Azevedo RA. Displasia fibrosa: osteoplastia com acesso Weber-Ferguson: relato de caso. Rev cir traumatol buco-maxilo-fac 2010 jan-mar ;10(1):

11. ALVES AL, Fernando C, Vilela DSA, Granato L, Próspero JD. Displasia fibrosa: relato de três casos. Rev Bras Otorrinolaringol 2002 mar.;68(2):288-92.

12. DOYON D, Cabanis EA, Frija J, Halimi P, Roger B. Tomografia computadorizada. 2. ed. Rio de Janeiro: Medsi; 2004.

13. HOFER M. Tomografia computadorizada: manual prático de ensino. 3. ed. Rio de Janeiro: Revinter; 2005.

14. SUTTON D. Tratado de radiologia e diagnostico por imagem. 6. ed. Rio de Janeiro: Revinter; 2003.

15. KANSKI JJ. Oftalmologia clínica: uma abordagem sistemática. 6. ed. Rio de Janeiro: Elsevier; 2008.

16. GUIMARÃES M. Anatomia e estrutura microscópica do nervo óptico. Minas Gerais: Hosp. Olhos 2003.

17. FERREIRA RGF, Santos MLB. Estudo morfológico e neurofuncional da cavidade orbital. Acta Sci Med 2012 5(1):2-8. 
NASCIMENTO PA, HENRIQUE S, HESS NETOA, OLIVIERI BV, RUFINO RA, TUCUNDUVA MJAPS. Avaliação da relação entre as dimensões da órbita e do forame óptico com as dimensões do nervo óptico pela tomografia computadorizada helicoidal. Rev. Odontol. Univ. Cid. São Paulo 2018 jan-mar; 30(1):54-63

ISSN 1983-5183

18. LIU X, Zhou C, Zhang G, Lin Y, Li S. [CT anatomic measurement of the optic canal and its clinical significance]. Zhonghua er bi yan hou ke za zhi 2000 Aug;35(4):275-7.

RECEBIDO EM 23/10/2017

ACEITO EM 23/10/2017 